\section{Uso de psicotrópicos entre universitários da área da saúde da Universidade Federal do Amazonas, Brasil}

\author{
Use of psychoactive drugs by health sciences \\ undergraduate students at the Federal University \\ in Amazonas, Brazil
}

\author{
Ana Cyra dos Santos Lucas 1 \\ Rosana Cristina Pereira Parente 2 \\ Neila Soares Picanço ${ }^{3}$ \\ Denis Alvaci Conceição 1 \\ Karen Regina Carim da Costa 1 \\ Igor Rafael dos Santos Magalhães 1 \\ João Cladirson Alves Siqueira ${ }^{1}$
}

\footnotetext{
1 Faculdade de Ciências da Saúde, Universidade Federal do Amazonas, Manaus, Brasil.

2 Instituto de Ciências Exatas, Universidade Federal do Amazonas, Manaus, Brasil. 3 Grupo de Pesquisa em Toxicologia, Universidade Federal do Amazonas, Manaus, Brasil.

Correspondência A. C. S. Lucas Departamento de Análises Clínicas e Toxicológicas, Faculdade de Ciências da Saúde, Universidade Federal do Amazonas. Rua Com. Alexandre Amorim 330, Manaus, AM 69053-030, Brasil. alucas@ufam.edu.br
}

\begin{abstract}
A survey was conducted with 521 undergraduate health sciences students from the Federal University in Amazonas, Manaus, Brazil. Lifetime alcohol consumption was reported by $87.7 \%$ students, as compared to $30.7 \%$ for tobacco, with the latter reported more frequently by males (39.7\%). The most common illicit drugs were solvents (11.9\%), marijuana (9.4\%), amphetamines and anxiolytics (9.2\% each), cocaine (2.1\%), and hallucinogens (1.2\%). The main reason for illicit drug use was curiosity. Lifetime use of anabolic steroids was reported by $2.1 \%$ of the students. Alcohol abuse in the previous 30 days was reported by $12.4 \%$ of the students. Events following drinking included: fights (4.7\%), accidents (2.4\%), classroom absenteeism (33.7\%), and job absenteeism (11.8\%). Another important finding was that $47.3 \%$ of students drove after drinking. Opinions on drug abuse and patterns agree with those from similar studies in other regions of Brazil.
\end{abstract}

Psychotropic Drugs; Health Occupations, Students; Drug Abuse

\section{Introdução}

O uso de substâncias psicotrópicas tem sido objeto de diversos estudos no Brasil, devido à crescente preocupação com os hábitos de consumo de drogas lícitas e ilícitas e seus impactos sociais, econômicos e, sobretudo, suas implicações na saúde da população. Além disso, medidas de prevenção são eficazes somente quando baseadas na realidade do consumo 1 , sendo importante a realização de pesquisas nos diversos segmentos da sociedade.

O destaque para os profissionais de saúde deve-se, por um lado, à sua responsabilidade na identificação e encaminhamento de pacientes com problemas relacionados ao uso de substâncias psicotrópicas 2 e, por outro, ao fato de servirem como modelo para seus pacientes 3 . Além disso, o fácil acesso e a fácil convivência com muitas dessas substâncias, aliados às condições de trabalho estressantes, tornariam esse grupo mais vulnerável ao abuso 4 .

Os estudos realizados no Brasil sobre uso de drogas entre estudantes universitários foram feitos principalmente no Sudeste 5,6,7 e Sul 8,9 do país, o mesmo ocorrendo com os estudos que enfocaram estudantes de escolas médicas 3,10 , 11,12. No Amazonas, o único trabalho realizado sobre o tema foi em Manaus, capital do Estado. Esse, entretanto, não abordou o consumo de drogas ilícitas, tratando apenas de uma das drogas lícitas: o tabaco ${ }^{13}$. Assim, o presente estu- 
do, ao levantar as informações de consumo, permite, além da comparação desse grupo de universitários com outros já descritos na literatura, fornecer subsídios aos futuros programas de prevenção voltados para os estudantes.

Este estudo teve como objetivos descrever os universitários da área da saúde quanto às características sócio-demográficas: sexo, idade e faixa etária; nível sócio-econômico; uso das drogas psicotrópicas lícitas e ilícitas; características do consumo e principais causas relacionadas a ele.

\section{Material e método}

A pesquisa foi realizada nos cursos de Farmácia, Medicina e Odontologia da Faculdade de Ciências da Saúde da Universidade Federal do Amazonas (UFAM), em três etapas, no período de 2002 a 2004. A população em estudo, portanto, foram alunos matriculados nesses cursos, assim distribuídos: alunos do 1o ao 11 o período do curso de Farmácia (180 alunos), alunos do 1o ao 12o período do curso de Medicina (484) e alunos do 1o ao 7o do curso de Odontologia (145).

Para determinação do tamanho da amostra foi levado em consideração: grau de confiabilidade de $95 \%$, precisão de $1,7 \%$ e prevalência de "usuários de droga na vida" de 0,10\%, com base nos resultados de uso de psicotrópicos obtidos dos trabalhos de Andrade et al. 11, Mesquita et al. 10, Boskovitz et al. 7, Barcellos et al. ${ }^{9} \mathrm{e}$ Kerr-Corrêa et al. ${ }^{3}$. A fórmula utilizada foi a de cálculo de tamanho de amostra para população finita 14 . O resultado foi uma amostra de 418 estudantes. A distribuição de amostra para os cursos foi feita levando-se em conta a proporção dos alunos de cada curso em relação ao total populacional. Dessa forma, deveriam ser amostrados 93 de Farmácia, 250 de Medicina e 75 de Odontologia.

A amostragem foi por conglomerados em dois estágios, sendo o primeiro os cursos e o segundo os períodos correspondentes ao semestre de realização da pesquisa. Em cada período sorteou-se uma disciplina e aplicou-se o questionário aos alunos que estavam matriculados na turma e presentes no dia da aplicação. Não tendo as disciplinas número igual de alunos matriculados, decidiu-se aplicar a todos os presentes que assinaram o Termo de Consentimento Livre e Esclarecido (TCLE), resultando em uma amostra de 521 estudantes, o que diminuiu a margem de erro para $1,29 \%$.

O instrumento utilizado foi um questionário padronizado autopreenchível e não identi- ficado, com 77 questões, dividido em cinco partes. A primeira explicava a pesquisa e apresentava o convite para dela participar. A segunda parte compreendia perguntas sobre dados sócio-demográficos. Na terceira, havia o questionamento sobre o uso de drogas, dividido em questões sobre tabaco, álcool, maconha, cocaína e derivados, medicamentos anfetamínicos, ansiolíticos, anticolinérgicos, barbitúricos, opióides, xaropes à base de codeína, solventes, alucinógenos, orexígenos, seguindo-se a classificação utilizada pelo Centro Brasileiro sobre Drogas Psicotrópicas (CEBRID) 15,16,17,18. Embora os anabolizantes não sejam drogas psicoativas, foram incluídos neste estudo devido ao seu potencial de abuso, como também questões sobre o uso de drogas injetáveis. A quarta parte do questionário consistia em um detalhamento sobre o uso abusivo de álcool, e a quinta, na indagação sobre o relacionamento entre os pais e os estudantes entrevistados, além da opinião destes sobre o uso das drogas.

O grupo envolvido com a coleta dos dados foi selecionado e treinado pela coordenadora do estudo. Fez-se prévio agendamento da turma/disciplina sorteada e nelas, após a apresentação dos objetivos do trabalho e obtenção do TCLE, os questionários eram distribuídos aos alunos para o preenchimento e a devolução era feita em uma urna à saída da sala.

Os questionários foram submetidos à supervisão para detecção de erros de negligência ou de interpretação e codificação das respostas, para a inserção dos dados em planilha Excel (Microsoft Corporation, Estados Unidos).

Para verificar as associações entre as diversas variáveis, hábito de consumo de drogas psicotrópicas, sexo, idade, estado civil, trabalho remunerado e nível sócio-econômico ${ }^{19}$, utilizou-se o teste do $\chi^{2}$, porém, quando algum valor esperado foi igual ou inferior a 5, optou-se pelo teste Exato de Fisher ou da razão de verossimilhança, segundo o caso. Para as prevalências também foram calculados os intervalos de confiança 95\%. O nível de significância estabelecido foi de 0,05 , empregando-se para a análise o programa SAS versão 6.12 (SAS Institute, Cary, Estados Unidos).

Em relação ao uso de drogas, empregou-se a classificação usada pela Organização Mundial da Saúde (OMS) 20 com relação à freqüência de uso da droga psicotrópica: "uso na vida" (quando a pessoa fez uso pelo menos uma vez na vida); "uso no ano" (quando a pessoa fez uso pelo menos uma vez nos 12 meses que antecederam a pesquisa); "uso no mês" (quando a pessoa fez uso pelo menos uma vez nos trinta dias que antecederam a pesquisa); "uso freqüente" 
(quando a pessoa fez uso seis ou mais vezes nos trinta dias que antecederam a pesquisa); e "uso pesado" (quando a pessoa fez uso vinte ou mais vezes nos trinta dias que antecederam a pesquisa).

A pesquisa foi aprovada pelo Comitê de Ética em Pesquisa com Seres Humanos da UFAM, por meio do processo 019/2003, e os respondentes assinaram termo de consentimento informado, de acordo com a Resolução n. 196/96 do Conselho Nacional de Saúde.

\section{Resultados}

O total de questionários aplicados foi de 540, dos quais apenas 19 foram devolvidos em branco $(3,5 \%)$, demonstrando a boa aceitação da pesquisa.

Dos alunos pesquisados, a maior representação foi de solteiros $(89,1 \%)$, do sexo feminino $(65,7 \%)$, da faixa etária de 19 a 21 anos $(36,1 \%)$, sem trabalho remunerado $(54,7 \%)$ e com nível sócio-econômico A (56\%) (Tabela 1).

Na Tabela 2 podem ser observados os dados de "uso na vida" das diversas drogas psicotrópicas, nos quais se nota que as mais utilizadas foram as legais. O "uso na vida" de álcool foi relatado por $87,7 \%$ dos estudantes (IC: 85,34 $90,06)$, não havendo diferença significativa entre o uso por estudantes do sexo feminino e masculino ( $\mathrm{p}=0,202)$. Para o "uso na vida" de tabaco, relatado por $30,7 \%$ dos estudantes (IC: 27,39-34,01), observou-se diferença significativa sendo maior o uso entre estudantes do sexo masculino (39,67\%; IC: 33,33-46,01; p = 0,001).

Entre as drogas psicotrópicas ilegais, as mais "usadas na vida" foram solventes (11,9\%; IC: 9,57-14,23), maconha (9,4\%; IC: 7,30-11,5), anfetamínicos $(9,2 \%$; IC: $7,12-11,28)$, cocaína $(2,1 \%$; IC: $1,07-3,13)$ e alucinógenos $(1,2 \%$; IC: $0,42-$ 1,98) (Tabela 2). Além disso, $2,1 \%$ dos estudantes pesquisados citaram "uso na vida" de esteróides anabolizantes. Houve diferença significativa entre os sexos, sendo maior o "uso na vi$d a$ " de maconha $(\mathrm{p}=0,001)$, solventes $(\mathrm{p}=0,001)$, anabolizantes e cocaína (ambos com $\mathrm{p}<0,001$ ) entre os homens.

Na Tabela 3 observa-se que os estudantes pertencentes ao nível sócio-econômico A apresentaram maior proporção de uso na vida para anfetamínicos (11,9\%; IC: 3,98-19,82), com significância estatística $(p=0,020)$. Embora sem significância estatística, observou-se ainda que os estudantes pertencentes ao nível sócio-econômico B apresentaram maior proporção de "uso na vida" para álcool (91,6\%; IC: 89,3793,83) e os pertencentes ao nível sócio-econô-

\begin{tabular}{|c|c|c|}
\hline \multicolumn{3}{|c|}{$\begin{array}{l}\text { Distribuição dos estudantes universitários da área } \\
\text { de saúde da Universidade Federal do Amazonas, } \\
\text { segundo as características sócio-econômicas } \\
\text { e sócio-demográficas, 2002/2004. }\end{array}$} \\
\hline \multirow[t]{2}{*}{ Característica } & \multicolumn{2}{|c|}{ Estudantes } \\
\hline & & $\%$ \\
\hline \multicolumn{3}{|l|}{ Curso } \\
\hline Farmácia & 162 & 31,1 \\
\hline Medicina & 264 & 50,7 \\
\hline Odontologia & 95 & 18,2 \\
\hline \multicolumn{3}{|l|}{ Sexo } \\
\hline Masculino & 188 & 35,8 \\
\hline Feminino & 337 & 65,7 \\
\hline \multicolumn{3}{|l|}{ Idade (anos) } \\
\hline$\leq 18$ & 59 & 11,5 \\
\hline $19-21$ & 185 & 36,0 \\
\hline $22-24$ & 165 & 32,2 \\
\hline$\geq 25$ & 104 & 20,3 \\
\hline \multicolumn{3}{|l|}{ Estado civil } \\
\hline Solteiro & 464 & 89,0 \\
\hline Casado & 50 & 9,6 \\
\hline Separado & 3 & 0,6 \\
\hline Outros & 4 & 0,8 \\
\hline \multicolumn{3}{|c|}{ Trabalho remunerado } \\
\hline Não & 284 & 54,7 \\
\hline Sim & 200 & 38,6 \\
\hline Às vezes & 35 & 6,7 \\
\hline \multicolumn{3}{|c|}{ Nível sócio-econômico } \\
\hline A & 269 & 56,0 \\
\hline$B$ & 131 & 27,3 \\
\hline C & 72 & 15,0 \\
\hline$D$ & 6 & 1,3 \\
\hline$E$ & 2 & 0,4 \\
\hline
\end{tabular}

mico D foram os que apresentaram maior proporção de "uso na vida" para solventes, maconha, cocaína e ansiolíticos (todos com 16,67\%).

A comparação da idade com que os estudantes usaram as drogas psicotrópicas pela primeira vez está mostrada na Tabela 4 , com os porcentuais sobre o total de estudantes que havia relatado "uso na vida" dessas substâncias. Observa-se que as faixas etárias com as maiores proporções de uso inicial da droga foram as de: 16 a 18 anos para álcool e tabaco; acima de 18 anos para ansiolíticos, solventes, anfetamínicos e cocaína; e para maconha foi igualmente dividida entre essas duas faixas. 
Tabela 2

Distribuição dos estudantes universitários da área de saúde da Universidade Federal do Amazonas, segundo o uso na vida de drogas psicotrópicas, segundo o sexo e as diferentes substâncias/classes farmacológicas, 2002/2004.

\begin{tabular}{|c|c|c|c|c|c|c|c|c|c|c|}
\hline \multirow[t]{2}{*}{ Droga } & \multicolumn{3}{|c|}{ Masculino } & \multicolumn{3}{|c|}{ Feminino } & \multirow[t]{2}{*}{$p$} & \multicolumn{3}{|c|}{ Total } \\
\hline & $\mathrm{n}$ & $\%$ & IC95\% & $n$ & $\%$ & IC95\% & & $\mathrm{n}$ & $\%$ & IC95\% \\
\hline Álcool & 165 & 90,16 & $87,87-92,45$ & 290 & 86,31 & $83,67-88,95$ & 0,202 & 455 & 87,7 & $85,34-90,06$ \\
\hline Alucinógenos & 4 & 2,17 & $0,00-11,93$ & 2 & 0,59 & $0,00-5,72$ & 0,192 & 6 & 1,2 & $0,42-1,98$ \\
\hline Anabolizantes & 10 & 5,43 & $0,00-16,64$ & 1 & 0,3 & $0,00-3,00$ & $<0,001$ & 11 & 2,1 & $1,07-3,13$ \\
\hline Anfetamínicos & 11 & 5,98 & $0,37-11,59$ & 37 & 10,98 & $3,58-18,38$ & 0,059 & 48 & 9,2 & $7,12-11,28$ \\
\hline Ansiolíticos & 16 & 8,7 & $2,03-15,37$ & 32 & 9,5 & $2,56-16,44$ & 0,763 & 48 & 9,2 & $7,12-11,28$ \\
\hline Anticolinérgicos & 2 & 1,09 & $0,00-13,13$ & 0 & 0,0 & $0,00-0,00$ & 0,124 & 2 & 0,4 & $0,00-0,85$ \\
\hline Barbitúricos & 2 & 1,09 & $0,00-9,60$ & 2 & 0,6 & $0,00-6,93$ & 0,617 & 4 & 0,8 & $0,16-1,44$ \\
\hline Cocaína & 10 & 5,49 & $0,00-16,75$ & 1 & 0,3 & $0,00-3,00$ & $<0,001$ & 11 & 2,1 & $1,07-3,13$ \\
\hline Maconha & 31 & 16,94 & $8,15-25,73$ & 18 & 5,36 & $0,08-10,64$ & 0,001 & 49 & 9,4 & $7,30-11,50$ \\
\hline Opióides & 2 & 1,09 & $0,00-10,92$ & 1 & 0,3 & $0,00-5,48$ & 0,287 & 3 & 0,6 & $0,05-1,15$ \\
\hline Orexígenos & 0 & 0,0 & $0,00-0,00$ & 1 & 0,3 & $0,00-9,27$ & 1 & 1 & 0,2 & $0,00-0,52$ \\
\hline Solventes & 34 & 18,48 & $10,40-26,56$ & 28 & 8,33 & $2,57-14,09$ & 0,001 & 62 & 11,9 & $9,57-14,23$ \\
\hline Tabaco & 73 & 39,67 & $33,33-46,01$ & 87 & 25,82 & $20,15-31,49$ & 0,001 & 160 & 30,7 & $27,39-34,01$ \\
\hline Xaropes & 0 & 0,0 & $0,00-0,00$ & 1 & 0,3 & $0,00-9,27$ & 1 & 1 & 0,2 & $0,00-0,52$ \\
\hline
\end{tabular}

Tabela 3

Distribuição dos estudantes universitários da área de saúde da Universidade Federal do Amazonas, segundo o uso na vida de drogas psicotrópicas, o nível sócio-econômico e as diferentes substâncias/classes farmacológicas, 2002/2004.

\begin{tabular}{|c|c|c|c|c|c|c|c|c|c|c|c|c|c|c|c|c|}
\hline \multirow[t]{2}{*}{ Droga } & \multicolumn{3}{|c|}{ Nível A } & \multicolumn{3}{|c|}{ Nível B } & \multicolumn{3}{|c|}{ Nível C } & \multicolumn{3}{|c|}{ Nível D } & \multicolumn{3}{|c|}{ Nível E } & \multirow[t]{2}{*}{$p$} \\
\hline & $n$ & $\%$ & IC95\% & $\mathrm{n}$ & $\%$ & IC95\% & $\mathrm{n}$ & $\%$ & IC95\% & $n$ & $\%$ & IC95\% & $\mathrm{n}$ & $\%$ & IC95\% & \\
\hline Álcool & 233 & 87,27 & $84,59-89,95$ & 120 & 91,60 & $89,37-93,83$ & 57 & 79,17 & $75,91-82,43$ & 5 & 83,33 & $80,34-86,32$ & 2 & 100 & - & 0,149 \\
\hline $\begin{array}{l}\text { Alucinó- } \\
\text { genos }\end{array}$ & 2 & 0,74 & $0,0-7,030$ & 3 & 2,29 & $0,00-13,26$ & 0 & 0,00 & $0,00-0,00$ & 0 & 0,00 & $0,00-0,00$ & 0 & 0 & - & 0,492 \\
\hline $\begin{array}{l}\text { Anaboli- } \\
\text { zantes }\end{array}$ & 6 & 2,23 & $0,00-9,89$ & 4 & 3,05 & $0,00-11,97$ & 0 & 0,00 & $0,00-0,00$ & 0 & 0,00 & $0,00-0,00$ & 0 & 0 & - & 0,416 \\
\hline $\begin{array}{l}\text { Anfetamí- } \\
\text { nicos }\end{array}$ & 32 & 11,90 & $3,98-19,82$ & 12 & 9,16 & $2,11-16,21$ & 1 & 1,39 & $0,00-4,25$ & 0 & 0,00 & $0,00-0,00$ & 0 & 0 & - & 0,02 \\
\hline Ansiolíticos & 25 & 9,29 & $2,11-16,47$ & 12 & 9,16 & $2,03-16,29$ & 6 & 8,33 & $1,50-15,16$ & 1 & 16,67 & $7,46-25,88$ & 0 & 0 & - & 0,94 \\
\hline $\begin{array}{l}\text { Anticoli- } \\
\text { nérgicos }\end{array}$ & 0 & 0,00 & $0,00-0,00$ & 2 & 1,53 & $0,00-15,76$ & 0 & 0,00 & $0,00-0,00$ & 0 & 0,00 & $0,00-0,00$ & 0 & 0 & - & 0,266 \\
\hline Barbitúricos & 3 & 1,12 & $0,00-11,08$ & 0 & 0,00 & $0,00-0,00$ & 0 & 0,00 & $0,00-0,00$ & 0 & 0,00 & $0,00-0,00$ & 0 & 0 & - & 0,478 \\
\hline Cocaína & 2 & 0,75 & $0,00-5,02$ & 5 & 3,85 & $0,00-13,36$ & 3 & 4,17 & $0,00-14,05$ & 1 & 16,67 & $0,00-35,10$ & 0 & 0 & - & 0,08 \\
\hline Maconha & 22 & 8,24 & $1,59-14,89$ & 16 & 12,21 & $4,29-20,13$ & 7 & 9,72 & $2,56-16,88$ & 1 & 16,67 & $7,66-25,68$ & 0 & 0 & - & 0,69 \\
\hline Opióides & 1 & 0,37 & $0,00-6,12$ & 2 & 1,53 & $0,00-13,15$ & 0 & 0,00 & $0,00-0,00$ & 0 & 0,00 & $0,00-0,00$ & 0 & 0 & - & 0,637 \\
\hline Orexígenos & 0 & 0,00 & $0,00-0,00$ & 1 & 0,76 & $0,00-15,00$ & 0 & 0,00 & $0,00-0,00$ & 0 & 0,00 & $0,00-0,00$ & 0 & 0 & - & 0,626 \\
\hline Solventes & 35 & 13,06 & $5,54-20,58$ & 13 & 9,92 & $3,25-16,59$ & 5 & 6,94 & $1,27-12,61$ & 1 & 16,67 & $8,35-24,99$ & 0 & 0 & - & 0,519 \\
\hline Tabaco & 77 & 28,62 & $22,53-34,71$ & 46 & 35,11 & $28,68-41,54$ & 23 & 31,94 & $25,65-38,23$ & 2 & 33,33 & $26,98-39,68$ & 0 & 0 & - & 0,516 \\
\hline Xaropes & 1 & 0,37 & $0,00-10,33$ & 0 & 0,00 & $0,00-0,00$ & 0 & 0,00 & $0,00-0,00$ & 0 & 0,00 & $0,00-0,00$ & 0 & 0 & - & 0,884 \\
\hline
\end{tabular}


Entre as drogas legais, encontrou-se que o "uso na vida" de tabaco foi maior $(\mathrm{p}=0,006)$ entre os estudantes que possuíam trabalho remunerado fixo ou esporádico (39\% e 28,57\%, respectivamente) frente aos $25,35 \%$ de "uso na vida" entre os que não tinham trabalho remunerado. O mesmo ocorreu com o uso na vida de ansiolíticos e anabolizantes (ambos $\operatorname{com} \mathrm{p}=$ 0,049).

Não foram encontradas associações estatisticamente significativas em relação ao trabalho remunerado e o uso na vida das demais substâncias pesquisadas ( $\mathrm{p}$ variando de 0,067 a 0,598), e tampouco para estado civil ( $p$ variando de 0,062 a 0,972); quanto à idade atual dos estudantes, houve significativo aumento do relato de "uso na vida" para tabaco $(\mathrm{p}=0,035)$, anfetamínicos $(p=0,034)$ e ansiolíticos $(p=0,034)$, sendo maior nas faixas acima de 22 anos (dados não apresentados).
Em relação ao "uso na vida" e ao convívio com outros usuários, variável estudada para álcool e tabaco, foi encontrada associação significativa para ambas as drogas. A proporção de "uso na vida" de tabaco foi maior $(\mathrm{p}=0,021)$ entre os estudantes que conviviam com outros fumantes na família $(35,77 \%)$ que entre os que não conviviam (26,37\%). O mesmo ocorre com o "uso na vida" de álcool, que é maior ( $\mathrm{p}=0,003$ ) entre os que convivem com bebedores $(90,18 \%)$ que entre os que não convivem com bebedores $(80,15 \%)$.

Na Tabela 5 estão apresentadas as opiniões dos estudantes pesquisados com relação ao uso de drogas psicotrópicas, divididas em tabaco, álcool, medicamentos sem receita médica e as drogas ilegais. Observa-se que as maiores proporções são para a opinião de que essas drogas "fazem muito mal à saúde", com exceção do álcool, sobre o qual a maioria dos estu-

Tabela 4

Distribuição dos estudantes universitários da área de saúde da Universidade Federal do Amazonas, segundo a idade em que ocorreu o uso inicial das drogas e as diferentes substâncias/classes farmacológicas, 2002/2004.

\begin{tabular}{|c|c|c|c|c|c|c|c|c|c|c|c|c|c|c|c|c|}
\hline \multirow[t]{2}{*}{ Droga } & \multicolumn{3}{|c|}{ Abaixo de 10 anos } & \multicolumn{3}{|c|}{ 10-12 anos } & \multicolumn{3}{|c|}{ 13-15 anos } & \multicolumn{3}{|c|}{ 16-18 anos } & \multicolumn{3}{|c|}{ Acima de 18 anos } & \multirow[t]{2}{*}{ Total } \\
\hline & $n$ & $\%$ & IC95\% & $\mathrm{n}$ & $\%$ & IC95\% & $\mathrm{n}$ & $\%$ & IC95\% & $\mathrm{n}$ & $\%$ & IC95\% & $\mathrm{n}$ & $\%$ & IC95\% & \\
\hline Icool & 11 & 3,70 & $1,91-6,83$ & 20 & 6,70 & $4,33-9,07$ & 77 & 25,80 & $21,65-29,95$ & 132 & 44,10 & $39,39-48,81$ & 59 & 19,70 & $15,93-23,47$ & 299 \\
\hline $\begin{array}{l}\text { Anfetamí- } \\
\text { nicos }\end{array}$ & 0 & 0,00 & $0,00-0,00$ & 1 & 2,90 & $0,00-7,62$ & 2 & 5,90 & $0,00-15,01$ & 10 & 29,40 & $16,59-42,21$ & 21 & 61,80 & $48,13-75,47$ & 34 \\
\hline Ansiolíticos & 0 & 0,00 & $0,00-0,00$ & 0 & 0,00 & $0,00-0,00$ & 2 & 5,10 & $0,00-13,04$ & 9 & 23,10 & $12,03-34,17$ & 28 & 71,80 & $59,98-83,62$ & 39 \\
\hline Barbitúricos & 0 & 0,00 & $0,00-0,00$ & 0 & 0,00 & $0,00-0,00$ & 0 & 0,00 & $0,00-0,00$ & 1 & 50,00 & $0,00-100,00$ & 1 & 50,00 & $0,00-100,00$ & 2 \\
\hline Cocaína & 0 & 0,00 & $0,00-0,00$ & 0 & 0,00 & $0,00-0,00$ & 1 & 11,10 & $0,00-27,28$ & 3 & 33,30 & $7,54-59,06$ & 5 & 55,60 & $28,44-82,76$ & 9 \\
\hline Maconha & 1 & 2,60 & $0,00-2,60$ & 1 & 2,60 & $0,00-6,78$ & 7 & 17,90 & $0,00-42,00$ & 15 & 38,50 & $25,72-51,28$ & 15 & 38,50 & $25,72-51,28$ & 39 \\
\hline Solventes & 0 & 0,00 & $0,00-0,00$ & 1 & 2,10 & $0,00-5,49$ & 6 & 12,50 & $0,00-30,44$ & 17 & 35,40 & $24,08-46,72$ & 24 & 50,00 & $38,16-61,84$ & 48 \\
\hline Tabaco & 8 & 6,50 & $2,87-11,21$ & 14 & 11,30 & $6,64-15,96$ & 31 & 25,00 & $0,00-55,75$ & 43 & 34,70 & $27,69-41,71$ & 28 & 22,60 & $16,44-28,76$ & 124 \\
\hline
\end{tabular}

Tabela 5

Distribuição dos estudantes universitários da área de saúde da Universidade Federal do Amazonas, segundo sua opinião sobre as diferentes drogas psicotrópicas.

\begin{tabular}{|c|c|c|c|c|c|c|c|c|}
\hline \multirow[t]{2}{*}{ Opinião } & \multicolumn{2}{|c|}{ Tabaco } & \multicolumn{2}{|c|}{ Álcool } & \multicolumn{2}{|c|}{$\begin{array}{c}\text { Medicamentos } \\
\text { sem receita }\end{array}$} & \multicolumn{2}{|c|}{$\begin{array}{c}\text { Outras } \\
\text { (drogas ilegais) }\end{array}$} \\
\hline & $n$ & $\%$ & $\mathrm{n}$ & $\%$ & $n$ & $\%$ & $n$ & $\%$ \\
\hline Faz muito mal à saúde & 360 & 69,6 & 190 & 36,8 & 284 & 55,0 & 434 & 83,9 \\
\hline Faz mal à saúde & 135 & 26,1 & 285 & 55,2 & 194 & 37,6 & 53 & 10,3 \\
\hline Não faz mal à saúde & 6 & 1,2 & 22 & 4,3 & 12 & 2,3 & 9 & 1,7 \\
\hline Não sei & 16 & 3,1 & 19 & 3,7 & 26 & 5,0 & 21 & 4,1 \\
\hline Total & 517 & 100,0 & 516 & 100,0 & 516 & 100,0 & 517 & 100,0 \\
\hline
\end{tabular}


dantes $(55,2 \%)$ opinou que apenas "faz mal à saúde". É interessante notar que as opiniões sobre as drogas estão igualmente distribuídas entre as opções "faz muito mal à saúde" e "faz mal à saúde”, exceto para as drogas ilegais que mais de $83,9 \%$ dos estudantes consideraram que "fazem muito mal à saúde".

Outras informações importantes foram obtidas com a pesquisa, como o motivo que fez os estudantes utilizarem as drogas psicotrópicas, com exceção de álcool e tabaco, sendo a mais relatada a curiosidade, constituindo o motivo para $72,9 \%$ dos que fizeram uso na vida. Também foi encontrado que $10,7 \%$ dos estudantes usaram as drogas psicotrópicas ilegais antes e $3,1 \%$ depois de ingressar na UFAM. E apenas um estudante $(0,2 \%)$ relatou o "uso na vida" de droga injetável, sem indicar a substância usada.

Do total de 521 estudantes pesquisados, $249(47,8 \%)$ relataram haver tomado bebidas alcoólicas até a embriaguez em algum momento da vida; $32(6,1 \%)$ o haviam feito num período de 1 a 5 dias no último mês; 21 (4\%), de $6 \mathrm{e}$ 19 dias no último mês e 15 (2,9\%), de 20 dias ou mais no último mês.

As bebidas preferidas foram a cerveja, relatada por 162 estudantes (31,8\%), e o vinho, relatado por $70(13,7 \%)$ estudantes. Entre os eventos ocorridos após beber, $4,7 \%$ do total de estudantes (8) indicaram haver brigado, 2,4\% (4) haviam sofrido algum acidente, $47,3 \%$ (80) haviam dirigido, $33,7 \%$ (57) faltado às aulas e $11,8 \%$ (20) faltado ao trabalho.

\section{Discussão}

Embora criticado por medir o "relato" e não o "consumo" verdadeiro de drogas, o emprego de questionários para levantamento de informações relacionadas ao uso de drogas ainda é o método mais usado por instituições nacionais e internacionais por ser de fácil aplicação, apresentar baixo custo, assegurar o anonimato e ter baixo índice de rejeição 20. Barros et al. 21 destacam que essas iniciativas são vistas pelos estudantes como mecanismo de prevenção e não de repressão, e que a grande aceitação de pesquisas desse tipo aumenta a confiabilidade nas respostas obtidas.

Os dados compilados com uma pesquisa dessa natureza são complexos, pois permitem o cruzamento de muitas variáveis. Para facilitar a análise dos resultados e a comparação com outros estudos, optou-se por destacar o "uso na vida" da droga, já que permite estimar o quanto a droga permeia a sociedade e seu potencial de uso por esta 22 , seguindo o padrão das publicações do CEBRID, por meio da Secretária Nacional Antidrogas (SENAD).

As drogas legais, álcool e tabaco, apresentaram respectivamente "uso na vida" de $87,7 \%$ e $30,7 \%$ entre os estudantes de saúde da UFAM. Esses valores são semelhantes aos trabalhos com estudantes de medicina de Kerr-Corrêa et al. 3 e Mesquita et al. 10, que variaram de $82 \%$ a $85,5 \%$ para o álcool e de $19,8 \%$ a $39 \%$ para o tabaco. Quando comparado aos dados do recente levantamento domiciliar sobre uso de drogas do CEBRID 1, observa-se que a população de estudantes universitários não difere muito, no "uso na vida" de álcool, da população em geral $(68,7 \%)$, tampouco no "uso na vida" de tabaco $(41,1 \%)$, embora apresente percentuais pouco maiores e menores, respectivamente.

Sendo o álcool e o tabaco as drogas de uso mais difundido na sociedade 1 , e até estimulado, principalmente no caso do álcool, não surpreende alta prevalência de seu uso pelos estudantes, embora estes, paradoxalmente, estejam conscientes dos seus efeitos prejudiciais, conforme se depreende da opinião de $92 \%$ dos estudantes sobre o álcool e de $95,7 \%$ sobre o tabaco. Esses estudantes se enquadram em um grupo que se sente protegido dos efeitos das drogas por possuírem conhecimento científico sobre as mesmas, como sugerido por Mesquita et al. 10 , revelando a necessidade de uma maior abordagem curricular sobre as conseqüências do uso dessas substâncias.

A prevalência de usuários do sexo masculino para o uso de tabaco, maconha, cocaína, solventes e anabolizantes está em concordância com a maioria dos outros estudos similares, como os de Boskovitz et al. 7 e Magalhães et al. 6, confirmando que a população masculina está mais propensa à experimentação dessas substâncias. A exceção a essa regra, também encontrada nos autores citados, é o "uso na vida" de anfetamínicos que foi maior entre estudantes do sexo feminino $(10,98 \%)$ que do masculino $(5,98 \%)$ nesta pesquisa, embora sem significância estatística.

Barcellos et al. 9 estudaram o padrão de uso de anfetaminas em universitários de várias áreas das ciências e demonstraram que, além da prevalência ser maior no sexo feminino, a finalidade do uso também difere entre os sexos: uso como estimulante e por recomendação de amigos entre os homens, e como anorexígeno e resultante de conversas com profissionais da saúde entre as mulheres.

Em relação às drogas psicotrópicas, com exceção de álcool e tabaco, as mais usadas na vida pelos estudantes da área da saúde da UFAM são, em ordem decrescente, os solven- 
tes, a maconha, os ansiolíticos e os anfetamínicos (empatados), a cocaína e os alucinógenos. A curiosidade aparece como o principal motivo apontado para o uso. Solventes e maconha também foram relatados nessa ordem por Mesquita et al. 10 e Kerr-Corrêa et al. ${ }^{3}$. A curiosidade também é apresentada por estes como o motivo principal, e ainda por Magalhães et al. 6 e Boskovitz et al. 7 .

Uma vez perguntados sobre o solvente utilizado, os 62 estudantes que relataram " $u$ so na vida" citaram o lança-perfume $(72,6 \%)$, a loló $(19,4 \%)$, a cola-de-sapateiro $(1,6 \%)$ e $6,5 \%$ não responderam. Magalhães et al. 6 já enfatizaram que a prevalência do uso de lança-perfume e loló pode ser devido à sua disponibilidade nas festividades do carnaval, e que a população universitária de renda mais elevada, como é a maioria no presente trabalho, apresenta menor tendência a usar os outros inalantes (thinner, acetona etc.), mais associados às rendas mais baixas.

$\mathrm{O}$ "uso na vida" encontrado para maconha, o segundo maior entre as drogas ilegais, toma grande importância devido à antiga discussão sobre o papel dessa droga na condução ao uso de outros tipos de psicotrópicos. Já foram encontrados dados que comprovaram que usuários universitários de maconha tiveram significativamente mais contato com outros tipos de droga que os não-usuários 6 .

Quanto à faixa etária do uso inicial das drogas, foi encontrada como principal a faixa de 16 a 18 anos para tabaco, álcool e maconha; e a de acima de 18 anos para anfetamínicos, solventes, barbitúricos e ansiolíticos. A comparação desses resultados é dificultada devido a faixas diferenciadas empregadas em outros trabalhos. Nota-se, porém, que pouco diferem dos encontrados por Magalhães et al. 6 e Mesquita et al. 10, em universitários de São Paulo, que apontaram as faixas de 15 a 19 anos para maconha e solventes, e acima de 20 anos para ansiolíticos e anfetaminas.

Para álcool e tabaco os mesmos autores indicaram a faixa de 10 a 14 anos como principal, portanto, com uso inicial em idades mais jovens que as encontradas no estudo em Manaus. Este resultado pode ser devido a diferenças culturais, que merecem ser mais bem investigadas entre os adolescentes dessas capitais.

Um exemplo de como os costumes das sociedades podem influenciar o consumo de drogas é apresentado por Ahmadi \& Javadpour 23 em seu estudo sobre estudantes universitários iranianos. Nele, foram encontrados $28 \%$ de " $u s o$ na vida" de tabaco, apenas $15 \%$ de álcool e 8,4\% de ópio, justificando-se estes achados por fatores culturais do país: o consumo de drogas (ex- ceto o tabaco) é totalmente inaceitável, porém há uma antiga tradição de usar o ópio para tratar de doenças mentais, além de uso recreativo.

$\mathrm{O}$ “uso na vida" de droga injetável foi aqui relatado por apenas $0,2 \%$ dos estudantes, abaixo do 1,5\% encontrado por Boskovitz et al. 7 , também entre universitários. Embora com baixo porcentual, é importante avaliar a ocorrência deste tipo de uso, visto que são associadas a ele várias doenças sexualmente transmissíveis, incluindo a AIDS.

A literatura apresenta dados contraditórios em relação à influência do ingresso na universidade sobre o uso de drogas. Plotnik et al. 12 citaram estudos que indicavam que o uso dessas substâncias aumenta após o ingresso no ambiente universitário, favorecido devido ao acesso mais fácil, ao menor controle social e à menor censura. Entretanto, Herrería \& Arancibia 24 e Brenes et al. 8 encontraram que a maioria dos universitários pesquisados havia experimentado psicotrópicos antes do ingresso, achado confirmado pelos presentes dados da UFAM.

Em relação aos eventos ocorridos com os estudantes após o uso de álcool, o mais citado foi dirigir, seguido de falta à escola, falta ao trabalho, envolvimento em brigas ou em algum acidente.

Minayo \& Deslandes 25 citaram estudos em que a participação de álcool chegava a 43-51\% dos casos de agressão, 40-50\% das mortes em acidentes de trânsito, e era a principal droga envolvida nos cerca de $13 \%$ de atendimentos hospitalares emergenciais relacionados com uso de drogas.

Considerando essas informações, é preocupante que um total de $12,4 \%$ dos universitários da área da saúde da UFAM tenham relatado alta freqüência no uso abusivo das bebidas alcoólicas (embriaguez), nos trinta dias que antecederam à pesquisa.

Sobre a opinião apresentada pelos estudantes devemos considerar que: são alunos da área da saúde, teoricamente com acesso às informações científicas sobre as substâncias, e que sofrem a influência da mídia sobre seus conceitos, como a maioria da população.

Segundo Noto et al. 26, a mídia brasileira é fonte de informações contraditórias e tendenciosas sobre as drogas psicotrópicas: as notícias veiculadas são caracterizadas por ênfase emocional, particularmente nos artigos publicados sobre as drogas ilegais, que são em maior número e com destaque a seus prejuízos para saúde.

Assim, a presença de propaganda abundante e sofisticada de estímulo ao uso de bebidas alcoólicas, as campanhas contra o tabaco e 
noção geral de que as drogas ilegais são mais danosas que as legais, justificam a maioria das opiniões encontradas neste trabalho sobre os psicotrópicos. A grande maioria conhece os efeitos prejudiciais do uso de álcool, 92\%, e, contraditoriamente, este é quase o mesmo percentual de "uso na vida" para essa substância $(87,7 \%)$. O maior percentual de alunos acha que as drogas ilegais "fazem muito mal à saúde", expressando a mesma opinião sobre o tabaco e os medicamentos sem receita médica. Magalhães et al. 6 também encontraram altos índices de desaprovação às drogas mesmo entre estudantes que já haviam usado drogas ilegais.

\section{Conclusão}

Os dados encontrados neste trabalho demonstraram que os estudantes da Faculdade de Ciên- cias da Saúde da UFAM apresentam um perfil de "uso na vida" de drogas psicotrópicas semelhante aos estudantes de outras regiões do Brasil.

Além disso, a opinião sobre as drogas e o padrão de uso dos estudantes tampouco diferem muito da população em geral, o que sugere pouco impacto da abordagem sobre o tema nos currículos acadêmicos atuais. Sendo, portanto, necessária maior inserção do tema drogas na formação acadêmica desses profissionais. A visão unicamente médico-terapêutica deve ser substituída por uma perspectiva multidisciplinar: farmacológica, humanística e social. Assim, serão formados profissionais de saúde com maior capacidade de compreender o fenômeno do uso de drogas e atuar adequadamente na sua prevenção, diagnóstico e tratamento.

\section{Resumo}

Foi realizado um levantamento sobre uso de psicotrópicos entre estudantes da Faculdade de Ciências da Saúde da Universidade Federal do Amazonas, Manaus, Brasil, em uma amostra de 521 alunos. O "uso na vida" de álcool foi relatado por $87,7 \%$ dos estudantes (IC95\%: 85,34-90,06) e o de tabaco por 30,7\% (IC 95\%: 27,39-34,01), sendo o último maior entre estudantes do sexo masculino (39,7\%; IC95\%: 33,33-46,01). As substâncias ilegais mais usadas foram: solventes (11,9\%; IC95\%: 9,57-14,23), maconha (9,4\%; IC95\%: 7,30-11,50), anfetamínicos e ansiolíticos (ambos com 9,2\%; IC95\%: 7,12-11,28), cocaína (2,1\%; IC95\%: 1,07$3,13)$ e alucinógenos $(1,2 \%$; IC95\%: 0,42-1,98). O principal motivo relatado para o uso de drogas ilegais foi a curiosidade. $O$ "uso na vida" de esteróides anabolizantes foi citado por $2,1 \%$ dos estudantes. O uso abusivo de álcool nos últimos 30 dias foi relatado por 12,4\% dos universitários. Entre os eventos ocorridos após a ingestão de bebidas alcoólicas, os estudantes citaram envolvimento em briga (4,7\%), acidentes (2,4\%), falta à escola (33,7\%), falta ao trabalho $(11,8 \%)$ e condução de veículos (47,3\%). A opinião sobre as drogas e o padrão de uso dos estudantes não diferem muito dos estudos semelhantes em outras regiões do Brasil.

Psicotrópicos; Estudantes de Ciências da Saúde; Abuso de Drogas

\section{Colaboradores}

A. C. S. Lucas realizou a revisão bibliográfica, planificação da aplicação do questionário, análise dos resultados, debates sobre os resultados e redação final. R. C. P. Parente participou da planificação da aplicação do questionário, análise estatística, análise dos resultados, debates sobre os resultados e redação final. N. S. Picanço e D. A. Conceição supervisionaram a aplicação do questionário e realizaram crítica e digitação dos dados e debates sobre os resultados. K. R. C. Costa, I. R. S. Magalhães e J. C. A. Siqueira realizaram revisão bibliográfica, aplicação dos questionários, crítica e digitação dos dados, análise de resultados e redação parcial.

\section{Agradecimentos}

Ao Conselho Estadual de Entorpecentes do Amazonas, Xerox do Brasil-Amazonas e Universidade Federal do Amazonas pelo apoio para a realização desta pesquisa, aos professores Francisco de Assis Costa de Lima e Maria Regina Marques Marinho pela revisão do texto, e às acadêmicas Walquíria de Castro Lima, Adriana Drumond Sardinha e Priscila de Almeida Lago, que participaram da aplicação do questionário. 


\section{Referências}

1. Carlini EA, Galduróz JCF, Noto AR, Nappo AS. I levantamento domiciliar sobre o uso de drogas psicotrópicas no Brasil - 2001. São Paulo: Centro Brasileiro sobre Drogas Psicotrópicas; 2002.

2. Borini B, Oliveira, CM, Martins, MG, Guimarães RC. Conceitos, concepções etiológicas e atitudes de estudantes de medicina sobre o uso e abuso de álcool. Correlações com os padrões de uso Parte 2. J Bras Psiquiatr 1994; 43:123-31.

3. Kerr-Corrêa F, Andrade AG, Bassit, AZ, Boccuto, NMVF. Uso de álcool e drogas por estudantes de medicina da Unesp. Rev Bras Psiquiatr 1999; 21:95-100.

4. Hughes H, Brandenburg N, Baldwin Jr. DC, Storr CL, Williams KM, Anthony JC, et al. Prevalence of substance use among physicians. JAMA 1992; 267:2333-9.

5. Gorenstein C, DeLucia R, Gentil V. Uso de psicoestimulantes e energizantes entre universitários. Rev Assoc Med Bras 1983; 29:45-6.

6. Magalhães MP, Barros RS, Silva MTA. Uso de drogas entre universitários: a experiência com a maconha como fator delimitante. Rev ABP-APAL 1991; 13:97-104.

7. Boskovitz EP, Cruz ETN, Neto FC, Moraes MS, Netto JVP, Ávila LA, et al. Uso de drogas entre estudantes universitários em São José do Rio Preto, São Paulo. Rev Bras Psiquiatr 1995; 22:87-93.

8. Brenes LFV, Hammes MF, Solé MTV, Hein R, Ramil KAA. Drogas ilícitas entre universitários. Rev AMRIGS 1986; 30:140-3.

9. Barcellos AP, Paggi AP, Silva DB, Campagnolo MI, Dieterich MDD, Santos RLR, et al. Padrão de consumo de anfetaminas entre universitários de Porto Alegre. Rev Psiquiatr Rio Gd Sul 1997; 19:161-9.

10. Mesquita AMC, Bucaretchi HA, Castel S, Andrade AG. Estudantes da Faculdade de Medicina da Universidade de São Paulo: uso de substâncias psicoativas em 1991. Rev ABP-APAL 1995; 17:47-54.

11. Andrade AG, Bassit AZ, Mesquita AM, Fukushima JT, Gonçalves EL. Prevalência do uso de drogas entre alunos da Faculdade de Medicina da Universidade de São Paulo (1991-93). Rev ABP-APAL 1995; 17:41-6.

12. Plotnik R, Azmus AD, Tannhauser M, Tannhauser SL. Utilização de psicotrópicos por estudantes universitários. Pesqui Med (Porto Alegre) 1986; 20:109-13.

13. Galvão JF, Carvalho FMS, Lucas ACS, Oliveira GMA, Mousinho MC. O hábito de fumar na área da saúde. II. Estudantes de medicina, farmácia e odontologia. Revista UA Série: Ciências da Saúde 1992; 2:1-19.

14. Domenéch-Massons JM. Métodos estadísticos en ciencias de la salud. Barcelona: Signo; 1998.
15. Carlini-Cotrim BHRS, Carlini EA, Silva Filho AR, Barbosa MTS. O uso de drogas psicotrópicas por estudantes de 1o e 2 o graus da rede pública estadual, em dez capitais brasileiras. Brasília: Centro de Documentação, Ministério da Saúde; 1987. (Série C: Estudos e Projetos)

16. Carlini EA, Carlini-Cotrim B, Silva Filho AR, Barbosa MTS. II Levantamento nacional sobre o uso de psicotrópicos em estudantes de primeiro e segundo graus - 1989. São Paulo: Centro Brasileiro sobre Drogas Psicotrópicas, Escola Paulista de Medicina; 1989

17. Galduróz JCF, D’Almeida V, Carvalho V, Carlini EA. III Levantamento nacional sobre o uso de psicotrópicos em estudantes de 1o e 2 o graus em 10 capitais brasileiras - 1993. São Paulo: Centro Brasileiro sobre Drogas Psicotrópicas, Escola Paulista de Medicina; 1994.

18. Galduróz JC, Noto AR, Carlini EA. IV Levantamento sobre o uso de drogas entre estudantes de 1o e 2 o graus em 10 capitais brasileiras - 1997. São Paulo: Centro Brasileiro sobre Drogas Psicotrópicas, Escola Paulista de Medicina; 1997.

19. Associação Brasileira dos Institutos de Mercado. Proposição para um novo critério de classificação socioeconômica. São Paulo: Associação Brasileira dos Institutos de Mercado; 1978.

20. Smart RG, Hughes PH, Jonhston LD, Anumonye A, Khant U, Medinamora ME, et al. A methodology for students drug-use surveys. Geneva: World Health Organization; 1980.

21. Barros RS, Almeida SP, Magalhães MP, Silva MTA. Idéias e imagens suscitadas em estudantes universitários numa pesquisa sobre drogas: uma contribuição ao trabalho preventivo. Boletim de Psicologia 1992; 42:15-26.

22. Centro Brasileiro de Informações sobre Drogas Psicotrópicas. Questão polêmica: para que serve sabermos sobre o uso na vida de uma droga? Boletim CEBRID 2004; 51:3.

23. Ahmadi J, Javadpour A. Evaluación del consumo de sustancias tóxicas entre los estudiantes de ciencias de la salud iraníes. European Journal of Psychiatry 2002; 16 (3 Suppl):181-4.

24. Herrería HV, Arancibia MV. Consumo de sustancias psicoativas en estudiantes universitarios. Revista de Psiquiatria (Chile) 1995; 1:17-25.

25. Minayo MCS, Deslandes SF. A complexidade das relações entre drogas, álcool e violência. Cad Saúde Pública 1998; 14:35-42.

26. Noto AR, Baptista MC, Faria ST, Nappo AS, Galduróz JCF, Carlini EA. Drogas e saúde na imprensa brasileira: uma análise de artigos publicados em jornais e revistas. Cad Saúde Pública 2003; 19:69-79.

Recebido em 18/Fev/2005

Versão final reapresentada em 29/Ago/2005

Aprovado em 27/Set/2005 